\title{
Pulse transition to similaritons in normally dispersive fibre amplifiers
}

\author{
K Hammani $^{{ }^{*}}$, S Boscolo ${ }^{2}$ and C Finot ${ }^{1}$ \\ ${ }^{1}$ Laboratoire Interdisciplinaire Carnot de Bourgogne, UMR 6303 CNRS - Université de \\ Bourgogne, 9 avenue Alain Savary, BP 47870, 21078 Dijon Cedex, France \\ ${ }^{2}$ Photonics Research Group, School of Engineering and Applied Science, Aston \\ University, Birmingham, B4 7ET, United Kingdom \\ *Now at Optoelectronics Research Centre, University of Southampton, Highfield, \\ Southampton, SO17 1BJ, United Kingdom \\ E-mail : christophe.finot@u-bourgogne.fr
}

\begin{abstract}
A detailed experimental characterization of the transition process of an initially Gaussian pulse to the asymptotic self-similar parabolic solution in optical fibre amplifiers operating in the normal dispersion regime is performed.
\end{abstract}

Keywords. Parabolic pulse, similariton, optical fibre amplifier.

\section{Introduction}

Optical pulses able to propagate in a self-similar manner in a nonlinear waveguide have generated a great deal of attention in the last decade [1-2]. A variety of smart examples taking advantage of the interaction of the longitudinally varying linear, non-linear and gain parameters of the waveguide have been reported [3-4] : for an accurate balance between these physical effects and a suitably chosen initial profile, the optical structure maintains its shape and is always a scaled version of itself, i.e., it evolves self-similarly. However, it was shown as early as 1993 that complex management of the longitudinal waveguide parameters is not mandatory for observing a self-similar behaviour [5]. Indeed, a parabolic intensity profile can evolve self-similarly in a passive normally dispersive fibre with constant parameters. Such a pulse acquires a monotonic (linear) instantaneous frequency shift or chirp as it propagates in the fibre, which ensures wave-breaking (WB)-free propagation in the presence of strong nonlinearity.

The intrinsic resistance of self-similar parabolic pulses to the deleterious effects of optical wave breaking has stimulated research in the field of high-power fibre amplifiers [6-7]. It was found that in the presence of linear amplification, these pulse solutions, so-called "similaritons", possess the remarkable property of being a global attractor to the system for arbitrary initial conditions [7-8]. This attracting 
nature of similariton solutions has been experimentally demonstrated in systems relying on amplification from either rare-earth doping (with ytterbium [7, 9] or erbium- [10-11] dopants) or Raman scattering [8, 12] as well as dispersion-decreasing fibres [13-14]. Numerous applications have benefited from the unique properties of parabolic pulses and their ability to be re-compressed with small substructures, ranging from high-power ultrashort pulse sources $[7,9,11]$ to mode-locked fibre lasers [15-16] and optical signal regeneration for high-bit rate telecommunications [17].

While the asymptotic similaritons features are well understood [7, 18], the physics of the transition to this solution from arbitrary initial pulses has not been fully explored. Most efforts have focused on a description with an initial parabolic pulse or with starting from a highly nonlinear regime of propagation [19-21]. In this work, we more focus on the adiabatic transition from a low-energy initial Gaussian profile to the self-similar parabolic state. The paper is organized as follows. In the first part of the article, we present preliminary numerical results that have helped us to design the experiment and identify the different regimes of pulse propagation that can take place in our Raman fibre amplifier. We then describe an experiment conducted in the absence of gain which confirms the transient parabolic-like pulse shaping that can occur in a passive fibre medium [22]. The experimental results recorded in the Raman amplifier configuration outline the reshaping process that the initial pulse undergoes throughout propagation in the gain fibre. The validity of the simple propagation model used - the nonlinear Schrödinger equation (NLSE) with a constant gain term, is discussed in the light of those experimental results.

\section{Preliminary numerical simulations}

\subsection{Model and parameters under investigation}

Pulse propagation in an optical fibre amplifier can be described with the NLSE with linear gain :

$$
i \frac{\partial \psi}{\partial z}-\frac{1}{2} \beta_{2} \frac{\partial^{2} \psi}{\partial t^{2}}+\gamma|\psi|^{2} \psi=i \frac{g}{2} \psi
$$

where $z$ denotes propagation distance, $t$ denotes time in a co-moving frame and $\psi(z, t)$ is the slowly varying pulse envelope of the electric field. The parameter $\beta_{2}$ is the second-order (group-velocity) dispersion (GVD) which is assumed to be normal. $\gamma$ is the Kerr nonlinearity parameter and $g$ is the distributed gain coefficient. We do not take into account here the effects of linear attenuation of the pump and pump depletion, so that the gain is assumed to be constant along the fibre. Moreover, for the temporal durations of the pulses being considered (typically, a few picoseconds) the frequency dependence of the gain can also be neglected.

The fibre parameters, chosen in accordance with the experiments, are: $\beta_{2}=3.3 \mathrm{ps}^{2} / \mathrm{km}, \gamma=1.7 / \mathrm{W} / \mathrm{km}$, and an average gain in the small-signal limit of $g=4 \mathrm{~dB} / \mathrm{km}$. An initial Gaussian temporal profile is wellsuited to obtain a clean parabolic shape in the fibre [23]. We therefore use a chirp-free Gaussian pulse with the full-width at half-maximum (FWHM) duration $T_{0}=6.8 \mathrm{ps}$ as the initial condition for our simulations and experiments. A wide range of input peak powers $P_{0}$ is tested.

\subsection{Pulse propagation regimes}

The initial pulse will undergo different dynamics in the fibre amplifier depending on its peak power and the propagation length. The results of numerical simulations of Eq. (1) that are summarized in figure 1a illustrate the different propagation regimes that can take place in the fibre. The regions in the parameter space corresponding to the three main pulse shaping processes observed are sketched in the schematic 
diagram of Fig 1b. To characterize the pulse shape here we use the parameter of misfit $M$ between the pulse temporal intensity profile $|\psi(t)|^{2}$ and a parabolic fit $\left|\psi_{P_{-} F I T}(t)\right|^{2}[19,22]$ of the same energy :

$$
M^{2}=\int\left[|\psi|^{2}-\left|\psi_{P_{-} F I T}\right|^{2}\right]^{2} d t / \int|\psi|^{4} d t
$$

The misfit factor is null for a perfect parabolic pulse whereas it is typically 0.13 for a Gaussian pulse.
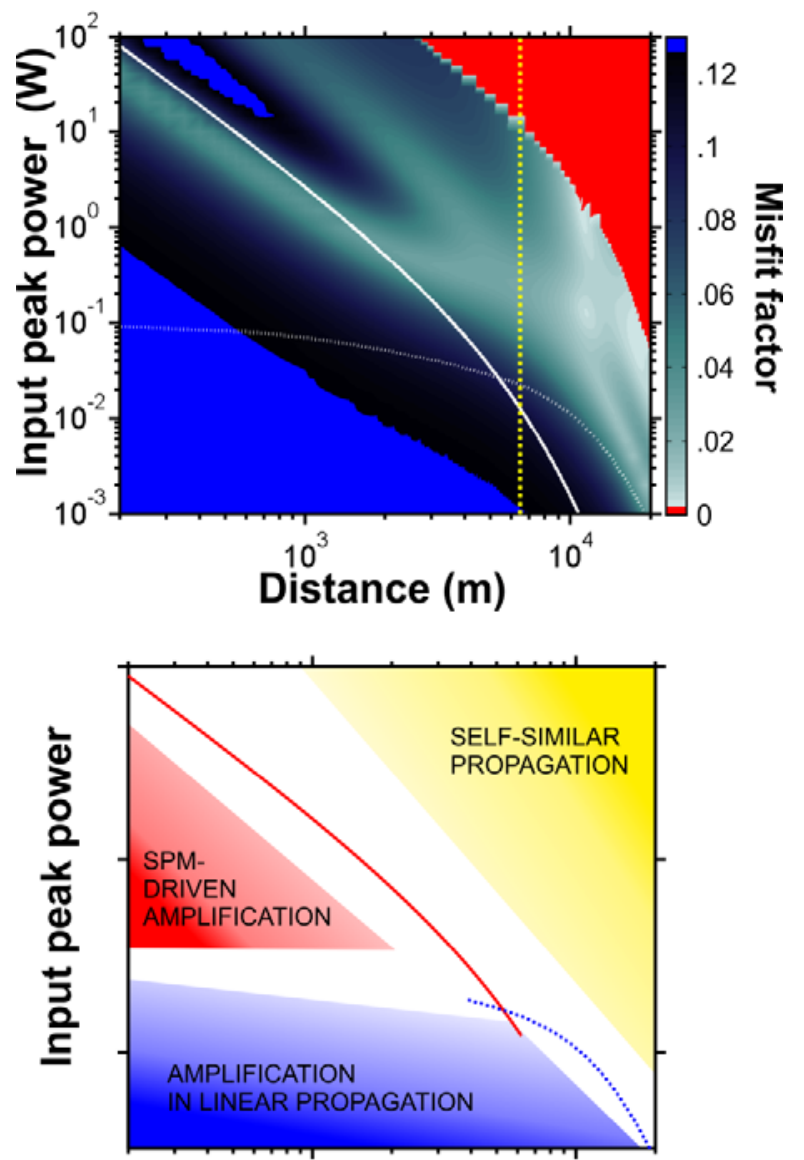

Distance

Figure 1. (a) Misfit parameter to a parabolic temporal shape versus propagation distance in the fibre amplifier and initial pulse peak power. The white dotted line is the linear propagation boundary of Eq. (6) and the white solid line is the transition distance to the parabolic pulse regime as given by the WB boundary of Eq. (4). The yellow solid line is the propagation length used in the experiment. The points where the M-factor equals 0.13 (i.e., the pulse shape is Gaussian) are shown in blue color, and the points where the $\mathrm{M}$-factor has not been computed (due to excessive requirements on the temporal window) are shown in red. (b) Schematic diagram of the different regimes of propagation displayed on a double logarithmic scale. The blue line is the linear propagation boundary of Eq. (6) and the red line is the transition distance to the parabolic pulse regime as given by the WB boundary of Eq. (4).

The pulse reaches the asymptotic similariton state at sufficiently large propagation distances. The misfit parameter approaches zero at large distances, showing that the intensity profile has become parabolic. In 
the self-similar regime, the root-mean square (rms) temporal duration $T_{S S}$ of the pulse evolves according to:

$$
T_{S S}(z)=\frac{3}{\sqrt{5}}\left(\sqrt{\frac{\pi}{\ln (2)}} \frac{T_{0} P_{0}}{4} \frac{\beta_{2} \gamma}{g^{2}}\right)^{1 / 3} \exp \left(\frac{g}{3} z\right)
$$

For high input powers, the dynamics is initially dominated by self-phase modulation (SPM) which introduces a chirp [19]. The interaction of SPM with GVD causes strong temporal reshaping of the pulse and ultimately leads to WB [24-25]. In a previous work [25], we have shown that the condition for the onset of WB in a gain fibre, relating the distance $z_{W B}$ where WB is first observed to the initial pulse power, provides an indication of the fibre length at which reshaping from a Gaussian pulse into a parabolic one occurs. This relationship is expressed in a dimensional form as :

$$
z_{\text {WB }}\left[\exp \left(z_{W B} g\right)-1\right]=\frac{1}{16 \ln (2) \exp (-3 / 2)} \frac{g}{\gamma \beta_{2}} \frac{T_{0}^{2}}{P_{0}}
$$

The analytical WB boundary of Eq. (4) is in good agreement with the numerical results for initial peak powers above a few hundreds $\mathrm{mW}$ (figure 1a, solid white line). Note however that the evolution of the misfit factor is in general non-monotonic in the SPM-driven amplification regime $[19,25]$ and the parabolic shape cannot be stabilized at this stage of propagation (i.e., the similariton regime has not been reached yet).

Dispersion is the major effect acting on the pulse for low input powers. In this regime of linear propagation, the initial Gaussian profile is preserved and, thus, the M-factor does not evolve (figure 1a). The pulse experiences significant temporal broadening, with its rms duration $T_{D}$ evolving according to a quadratic law:

$$
T_{D}(z)=\frac{T_{0}}{2 \sqrt{2 \ln (2)}} \sqrt{1+\left(4 \ln (2) z \frac{\beta_{2}}{T_{0}^{2}}\right)^{2}}
$$

Large propagation distances in the amplifier are required for the pulse to evolve from the linear to the self-similar regime. Note that the transition process between these two regimes is strictly adiabatic with a continuous decrease of the misfit factor along the propagation distance. For a given input power, a boundary for the linear propagation regime can be set at the distance at which the temporal duration $T_{D}$ and peak power of the pulse equate the pulse duration $T_{S S}$ and peak power in the self-similar regime, leading to the following implicit equation for the propagation length :

$$
\exp \left(\frac{-g}{3} z\right) \sqrt{1+\left(4 \ln (2) z \frac{\beta_{2}}{T_{0}^{2}}\right)^{2}}=6 \sqrt{\frac{2 \ln (2)}{5}}\left(\sqrt{\frac{\pi}{\ln (2)}} \frac{P_{0}}{4 T_{0}^{2}} \frac{\beta_{2} \gamma}{g^{2}}\right)^{1 / 3}
$$

The theoretical predictions from Eq. (6) are in good agreement with the numerical results (figure 1a, white dotted line). Note however that the Gaussian and self-similar pulses in general exhibit different levels of chirp at the border between the two regions.

This insight into the different propagation regimes taking place in the fibre amplifier provides a guideline for the choice of the initial pulse power and fibre length to deploy in the experiment. To observe the transition from the linear amplification regime to the self-similar regime, in this work we vary the initial pulse peak power. It would seem more natural to perform a study along the length of propagation, using a cut-back measurement procedure for example. However, this method is very difficult to 
implement in practice and to interpret due to the effects of pump depletion that can be significant in the counterpropagating pumping configuration used here. The fibre length is chosen in accordance with the range of input peak powers that can be achieved and characterized. A power range between the milliwatt and Watt levels suggests a length of $6.2 \mathrm{~km}$ to observe a clear transition of the pulse to the asymptotic similariton state. The map of figure 1a will therefore be scanned along the vertical yellow line.

\section{Experimental set-up}

To illustrate the transition dynamics, we analyze a 6.2-km long (cf. Section 2) Raman fibre amplifier operating at normal dispersion and using initial low-energy Gaussian pulses at $1552 \mathrm{~nm}$. The experimental setup is sketched in figure 2 . The pulses are delivered by a mode-locked erbium-doped fibre laser. The initial picosecond pulses have an hyperbolic secant profile. By using an adequate spectral transfer function provided by an optical spectral shaper based on the liquid crystal on silicon technology [26], we shaped the intensity spectrum of the pulses into a Gaussian profile with a FWHM spectral width of $67 \mathrm{GHz}$ (see inset of figure 2, black solid line). The resulting pulses have a temporal duration of $6.8 \mathrm{ps,}$ which yields a time-bandwidth product nearly equalizing the transform limit for a Gaussian pulse (0.44). The 10-GHz pulse stream is then modulated by a periodic sequence of alternating 0 's and 1's to decrease the pulse repetition rate and obtain a higher peak power for the same average power. Lowering the repetition rate also allows to limit the effects of pump depletion and gain saturation. A high-power erbium-doped fibre amplifier (EDFA) boosts the pulse peak power up to the Watt level, and then a variable attenuator finely controls the level of peak power launched in the gain fibre.

The Raman amplifier is based on a non-zero dispersion shifted fibre (NZ-DSF) having the parameters described in Section 2. A counterpropagating pumping scheme is favoured to avoid temporal depletion of the pulses [12] and limit the transfer of intensity noise from the pump to the amplified signal [27]. The pump is a partially coherent laser source with a 40-GHz spectral linewidth, operating at 1455 $\mathrm{nm}$ and delivering an average power of $2 \mathrm{~W}$ high-power wavelength-division multiplexing couplers are used to combine or separate the pump and the signal.

An ultrafast optical sampling oscilloscope (OSO) [28] with a picosecond temporal resolution (EXFO PSO device) is used at the output of the fibre to record the fine details of the temporal structure of the pulses. In contrast to the frequency-resolved optical gating (FROG) technique used in [7, 10, 12], an OSO allows to directly retrieve the pulse temporal profile (without any deconvolution). From a practical viewpoint, the OSO's flexibility enables systematic experimental studies with large sets of data. Moreover, a second-harmonic-generation FROG requires relatively high peak powers and, thus, is not suitable for the characterization of low-power pulses as the ones that are studied in this work.

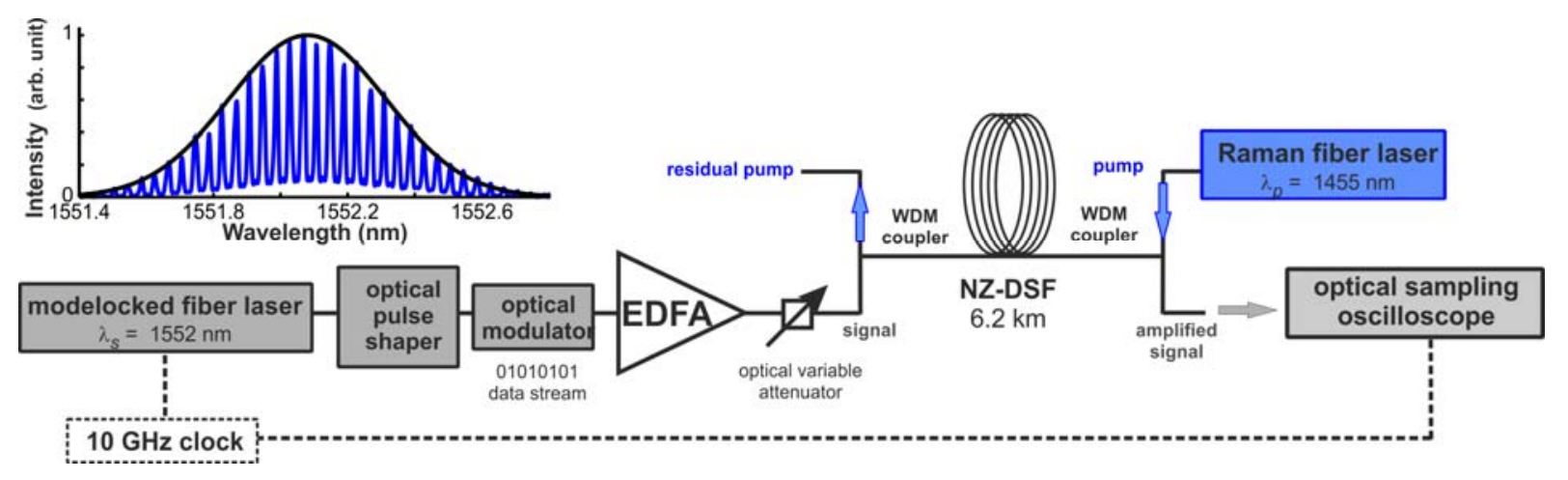


Figure 2. Schematic of the experimental setup. Top left inset : Measured optical spectrum of the signal entering the NZ-DSF (blue curve). Also shown is a Gaussian fit with a FWHM width of $67 \mathrm{GHz}$ (solid black curve).

\section{Pulse dynamics in passive fibre}

In this section, we describe the experimental results obtained for pulse propagation in the NZ-DSF in the absence of gain. Figure 3 shows the temporal intensity profile of the output pulse from the fibre for varied initial peak powers. It is seen that for low input powers, the output pulse remains Gaussian in shape. For input peak powers above $200 \mathrm{~mW}$, the output pulse shape becomes close to a parabola. This parabolic shape is not maintained with further increase of the input power, and the top of the intensity profile becomes flat. This is fully consistent with the transient pulse evolution preceding WB predicted in [24] and experimentally validated in [22], and confirms that parabolic pulses generated in a passive fibre have a finite life distance that depends sensibly on the initial conditions. Note that the propagation distance under investigation is not large enough to observe a long-term far-field evolution and formation of spectronic self-similar pulses, as it is discussed in [29-30].
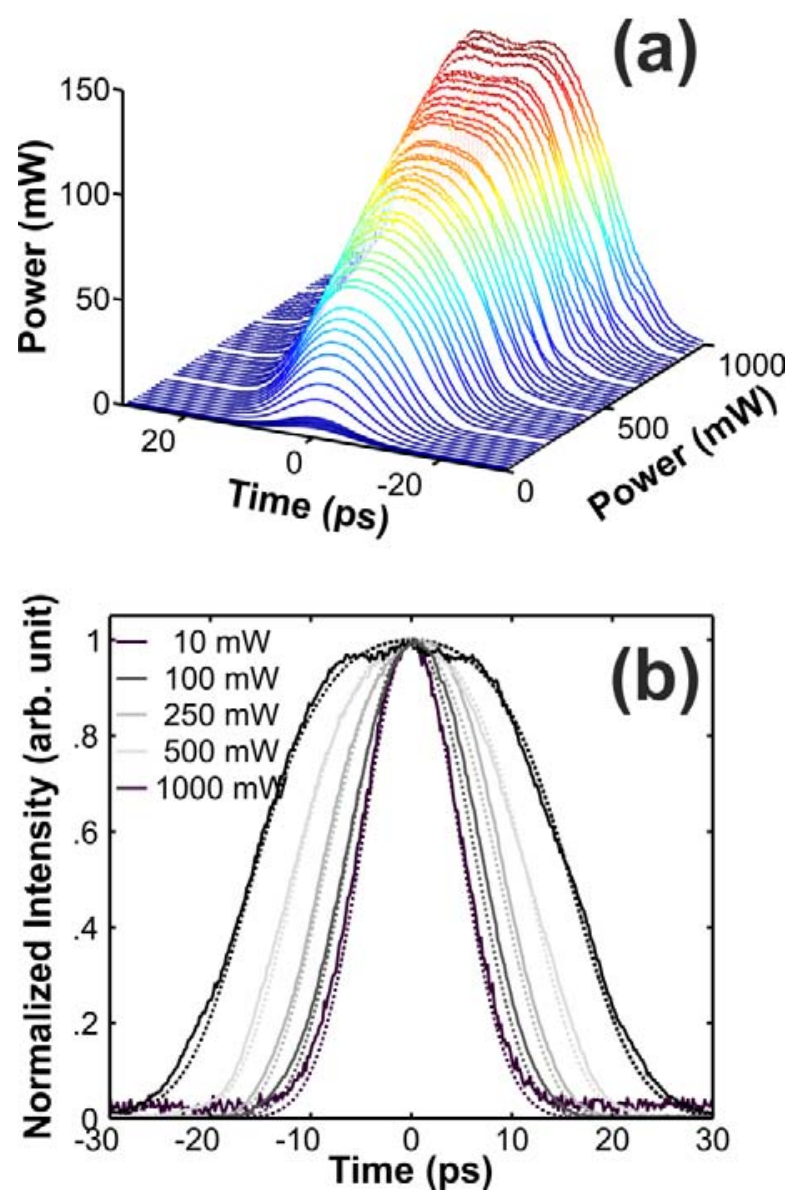
Figure 3. (a) Experimental temporal intensity profile of the output pulse from the passive fibre for varied initial peak powers. (b) Temporal profiles for a set of selected input peak powers. Solid curves: experimental results, dashed curves: results of numerical integration of the NLSE (Eq. (1) without gain).

The rms pulse duration and misfit parameter to a parabolic temporal shape at the end of the passive fibre computed from the experimental data are plotted in figure 4 and compared with the results of the numerical integration of Eq. (1) without gain. Note also that approximate analyses based on the method of moments have recently been reported for describing the nonlinear evolution of optical pulses in nonlinear fibres with normal GVD [31-32]. Both the rms duration and shape factor variations with input power observed experimentally are accurately reproduced by the numerical simulations. The agreement between the experimental and numerically computed intensity profiles in figure $3 \mathrm{~b}$ is also excellent. This further validates the use of the NLSE propagation model in a passive fibre, and also indicates that the input data from the model such as the initial pulse and the fibre parameters, are conveniently characterized.

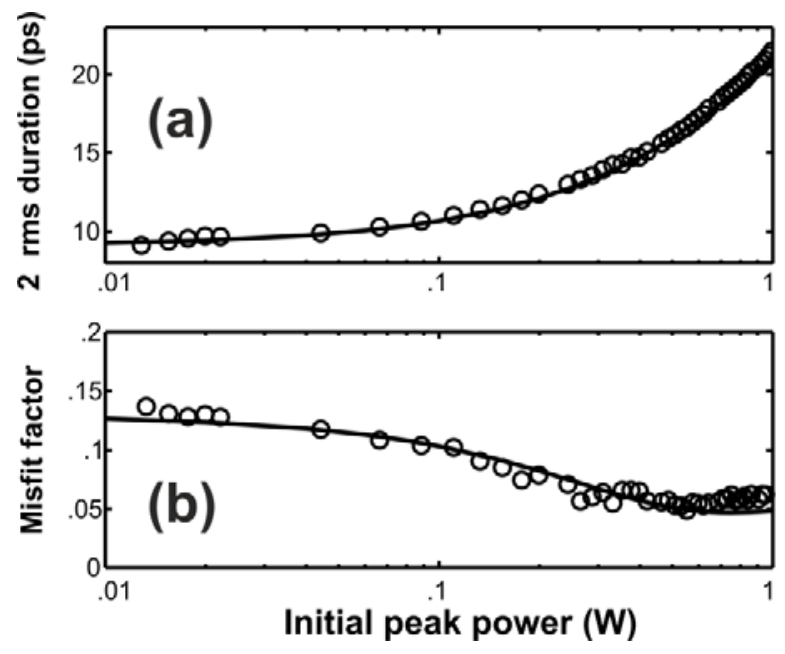

Figure 4. (a) rms temporal width of the pulse and (b) misfit parameter to a parabolic temporal shape at the end of the passive fibre for varied initial peak powers. The experimental results (circles) are compared with the results of numerical integration of the NLSE (Eq. (1) without gain, solid black curves)

\section{Pulse dynamics in fibre amplifier}

Here we experimentally characterize the pulse dynamics in the Raman fibre amplifier. Experimental temporal intensity profiles recorded for varied initial peak powers are summarized in figure 5 . Results plotted on a linear scale clearly exhibit the transition from a low-power Gaussian pulse affected mainly by dispersion towards a parabolic self-similar pulse. In contrast to the passive propagation described in the previous section, now the formed parabolic shape is preserved with increasing initial power, thus confirming the attracting nature of the amplifier similariton solution. Results plotted on a semilogarithmic scale (inset of figure 5b) show the rapidly falling leading and trailing edges characteristic of the parabolic form, in agreement with previous experimental observations [7-8]. We could argue the presence of low-amplitude wings about the central parabolic core region (indicated by a blue arrow), which are associated to the intermediate asymptotic regime of the self-similar propagation $[18,20]$. 
However, the dynamic range of our ultrafast characterization device is not sufficiently high to provide a clear conclusion on this issue, contrary to using FROG combined with adequate data treatment [10].
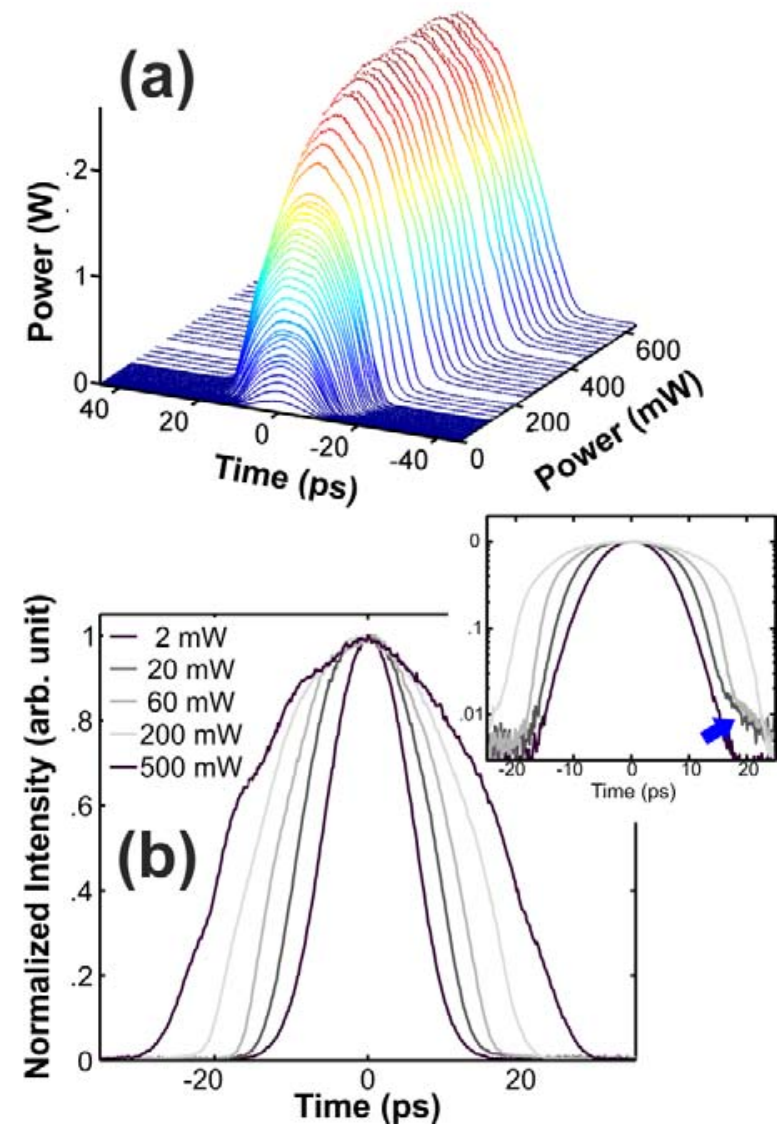

Figure 5. (a) Experimental temporal intensity profile of the output pulse from the Raman fibre amplifier for varied initial peak powers. (b) Temporal profiles for a set of selected input peak powers. Inset: temporal profiles plotted on a semi-logarithmic scale.

Figure 6 shows the variation of the rms temporal duration of the output pulse from the amplifier with the initial peak power. For input powers below $10 \mathrm{~mW}$, a linear dispersive pulse evolution takes place in the amplifier, as indicated by the very small power-dependence of the pulse width. The linear propagation regime is followed by a regime where the rms width scales exponentially with the input peak power, as shown by the good agreement between the experimental data and a linear fit plotted on a double logarithmic scale (grey dashed line). While this behaviour is qualitatively consistent with the predictions from Eq. (3) for the temporal duration of the asymptotic similariton solution (red dashed line), the slope of the experimental curve is significantly smaller than that of the similariton state curve. The results of the numerical integration of Eq. (1) (blue solid line) confirm that the propagation model based on the NLSE with a constant gain overestimates the temporal broadening that the pulse undergoes upon propagation in the fibre. This is also evident from the comparison of the temporal intensity profiles in Fig 5a with those in the inset of figure 6. 
In order to achieve quantitative agreement between theoretical and experimental results, we have included the longitudinal evolution of the gain in the numerical model. Such an evolution takes into account two physical effects : the progressive attenuation of the pump due to the linear loss of the fibre a nearly 2- $\mathrm{dB}$ attenuation is undergone by the pump between the fibre input and output in our configuration -, and the progressive saturation of the gain. The latter effect has been assessed through experimental measurements : seeding the amplifier with a milliwatt peak power signal yielded an integrated gain of $25 \mathrm{~dB}$, whereas the gain decreased down to $14 \mathrm{~dB}$ for a Watt-power-level input signal. Numerical simulations including the longitudinal variation of the gain reproduce accurately the experimental results in figure 6 (solid black curve). It is worth noting here that the asymptotic similariton solution is found for any longitudinal gain profile in a fibre amplifier [12, 33], and the functional form of the gain profile determines only the self-similar scaling of the propagating pulse in the amplifier. It has also been demonstrated that gain saturation does not affect the overall parabolic profile of the similariton [34-35].

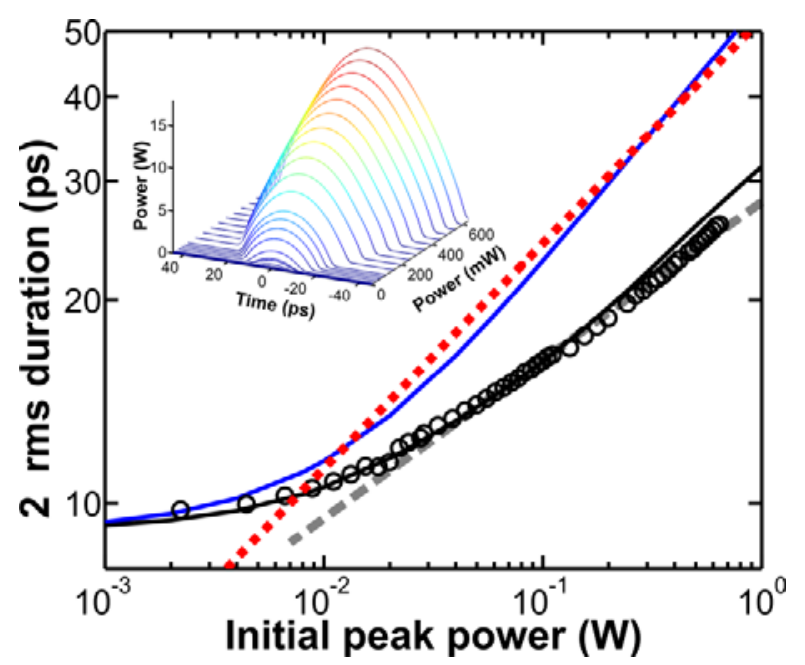

Figure 6. Rms temporal width of the output pulse from the Raman fibre amplifier for varied initial peak powers. Circles: experimental results, grey dashed line: linear (on double logarithmic scale) fit to the experimental data, blue solid curve: results of numerical integration of Eq. (1), red dashed line: analytical predictions from Eq. (3) (red dashed line). . The inset shows the evolution of the output pulse intensity profile as obtained from numerical integration of Eq. (1). Numerical results taking into account the longitudinal variation of the gain are plotted as a solid black curve.

The numerical model including longitudinally varying gain can also finely reproduce the experimentally measured temporal intensity profile of the output pulse from the amplifier, as the comparison of figure $7 \mathrm{a}$ with figure $5 \mathrm{~b}$ shows. Furthermore, the variation of the misfit parameter to a parabolic temporal shape with the input power (figure 7b) confirms that reshaping of the initial pulse to the parabolic self-similar form occurs in the amplifier for input peak powers above $100 \mathrm{~mW}$. Misfit factors as low as 0.03 are recorded, which are significantly smaller than those obtained in the passive fibre configuration (Section 3). We also note that the evolution of the misfit factor with varying input peak power as computed from both experiment and numerical simulation is not strictly monotonic. Similar non-monotonic behaviour has been reported in previous studies of the longitudinal evolution of this metric along a fibre amplifier [19, 25]. 

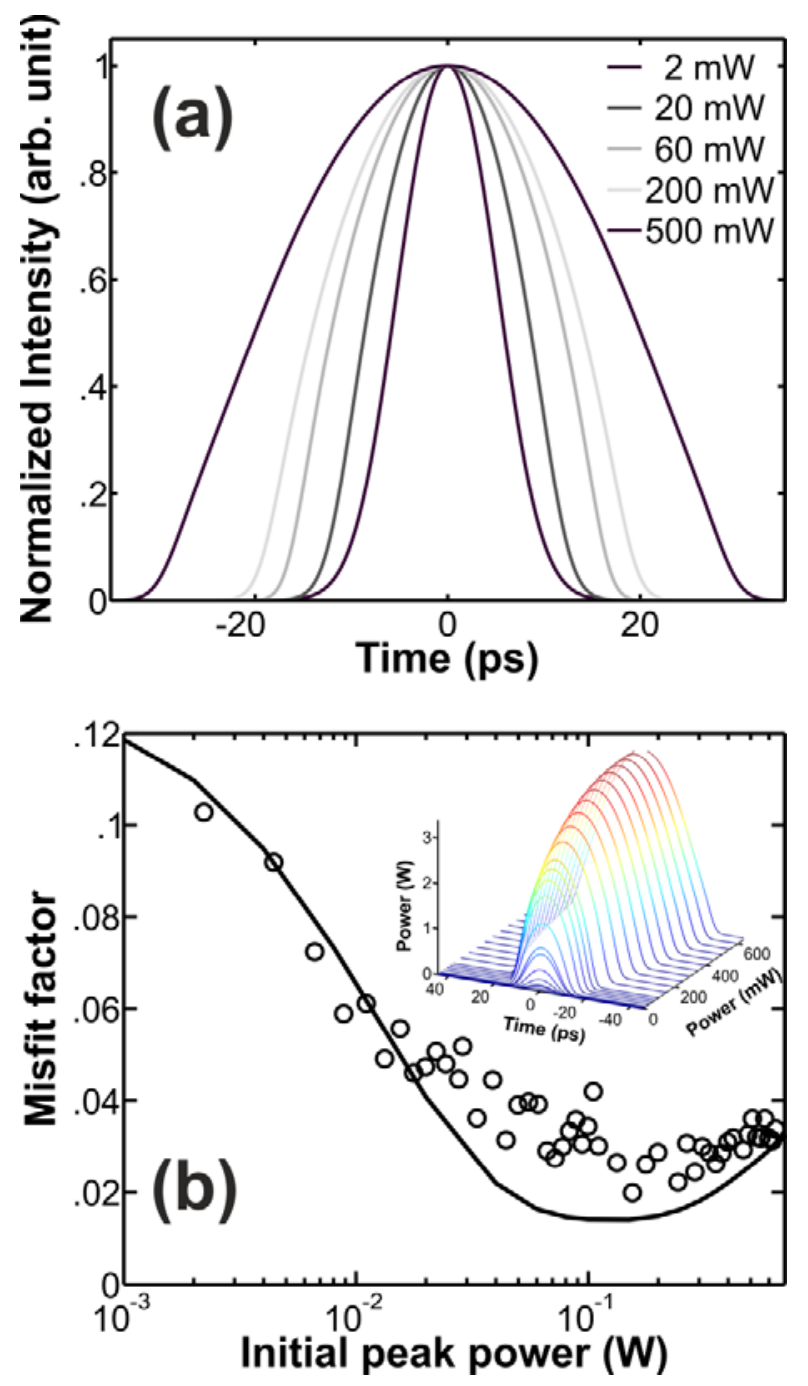

Figure 7. (a) Temporal intensity profile of the output pulse from the Raman fibre amplifier for a set of initial peak powers as obtained by the numerical model including the longitudinal gain variation. (b) Misfit parameter to a parabolic temporal shape at the amplifier output for varied initial peak powers. The experimental results (circles) are compared with the results of numerical simulations taking into account the varying gain (solid curve). The inset shows the numerically computed evolution of the output pulse intensity profile.

\section{Conclusion}

We have presented a detailed experimental characterization of the adiabatic transition process of an initially low-energy Gaussian pulse to the asymptotic self-similar parabolic solution in a Raman fibre amplifier. The experimental study coupled with a numerical analysis highlights the various stages of the nonlinear reshaping. Furthermore, the impact of saturation of the gain in the amplifier is also clearly shown, requiring the inclusion of a varying gain along the fibre in the numerical model. The OSO-based pulse characterization technique used in this work can be advantageously employed to measure other 
advanced pulse shapes generated through nonlinear pulse reshaping in normally dispersive fibres, such as triangular [36] and rectangular pulses [37]. The results presented in this paper demonstrate that even though optical amplifier similaritons have been highlighted as soon as 2000, there are still some aspects of the nonlinear pulse dynamics that are not fully explored. Many applications such as optical signal processing and mode-locked fibre lasers, may benefit from this a better understanding.

\section{Acknowledgement}

We thank Brandon G. Bale for initial discussions which have stimulated this experimental research work. We also thank Julien Fatome for experimental support.

This work has benefited from the PICASSO platform, and has been supported by the Conseil Regional de Bourgogne (PhotCom Pari scheme). We also acknowledge the French Ministry and the British Council for supporting the collaboration between the authors' institutions through the Alliance funding scheme.

\section{References}

[1] Dudley J M, et al. 2007 Self-similarity in ultrafast nonlinear optics Nat. Phys. 3 597-603

[2] Segev M, Soljacic M, and Dudley J M 2012 Fractal optics and beyond Nat Photon 6 209-210

[3] Kruglov V I, Peacock A C, and Harvey J D 2003 Exact self-similar solutions of the generalized nonlinear Schrödinger equation with distributed coefficients Phys. Rev. Lett. 90113902

[4] Ponomarenko S A and Agrawal G P 2006 Do solitonlike self-similar waves exist in nonlinear optical media ? Phys. Rev. Lett. 97013901

[5] Anderson D, et al. 1993 Wave-breaking-free pulses in nonlinear optical fibers J. Opt. Soc. Am. B $101185-1190$

[6] Finot C, et al. 2009 Optical parabolic pulse generation and applications IEEE J. Quantum Electron. 45 1482-1489

[7] Fermann M E, et al. 2000 Self-similar propagation and amplification of parabolic pulses in optical fibers Phys. Rev. Lett. 84 6010-6013

[8] Finot C, Millot G, and Dudley J M 2004 Asymptotic characteristics of parabolic similariton pulses in optical fiber amplifiers Opt. Lett. 29 2533-2535

[9] Dupriez P, et al. 2006 High-power, high repetition rate picosecond and femtosecond sources based on Yb-doped fiber amplification of VECSELS Opt. Express 14 9611-9616

[10] Billet C, et al. 2005 Intermediate asymptotic evolution and photonic bandgap fiber compression of optical similaritons around $1550 \mathrm{~nm}$ Opt. Express 13 3236-3241

[11] Tamura K and Nakazawa M 1996 Pulse compression by nonlinear pulse evolution with reduced optical wave breaking in erbium-doped fiber amplifiers Opt. Lett. 21 68-70

[12] Finot C 2005 Influence of the pumping configuration on the generation of optical similaritons in optical fibers Opt. Commun. 249 553-561

[13] Hirooka T and Nakazawa M 2004 Parabolic pulse generation by use of a dispersion-decreasing fiber with normal group-velocity dispersion Opt. Lett. 29 498-500

[14] Finot C, et al. 2007 Parabolic pulse generation with active or passive dispersion decreasing optical fibers Opt. Express 15 15824-15835

[15] Ilday F Ö, et al. 2004 Self-similar evolution of parabolic pulses in a laser Phys. Rev. Lett. 92 213902

[16] Oktem B, Ulgudur C, and Ilday F Ö 2010 Soliton-similariton fibre laser Nat. Photonics 4 307311

[17] Finot C, et al. 2011 Active Mamyshev regenerator Optical Review 18 257-263 
[18] Boscolo S, et al. 2002 Self-similar parabolic optical solitary waves Theor. Math. Phys. 133 16471656

[19] Finot C, et al. 2006 Parabolic pulse evolution in normally dispersive fiber amplifiers preceding the similariton formation regime Opt. Express 14 3161-3170

[20] Kruglov V I, et al. 2002 Self-similar propagation of parabolic pulses in normal-dispersion fiber amplifiers J. Opt. Soc. Amer. B 19 461-469

[21] Wabnitz S 2007 Analytical Dynamics of parabolic pulses in nonlinear optical fiber amplifiers IEEE Photon. Technol. Lett. 19 507-509

[22] Finot C, et al. 2007 Parabolic pulse generation through passive nonlinear pulse reshaping in a normally dispersive two segment fiber device Opt. Express 15 852-864

[23] Ozeki Y, et al. Clean similariton generation from an initial pulse optimized by the backward propagation method. in Conference on Lasers and Electro-Optics (CLEO US). 2004: OSA Trends in Optics and Photonics Series.

[24] Anderson D, et al. 1992 Wave-breaking in nonlinear optical fibers J. Opt. Soc. Am. B 9 13581361

[25] Finot C, et al. 2008 Beneficial impact of wave-breaking on coherent continuum formation in normally dispersive nonlinear fibers J. Opt. Soc. Am. B 25 1938-1948

[26] Clarke A M, et al. 2010 Reconfigurable Optical Pulse Generator Employing a Fourier-Domain Programmable Optical Processor J. Lightw. Technol. 28 97-103

[27] Hammani K, Finot C, and Picozzi A 2011 Extreme statistics in Raman fiber amplifier : from analytical description to experiments Opt. Commun. 284 2594-2603

[28] Andrekson P A and Westlund M 2007 Nonlinear optical fiber based high resolution all-optical waveform sampling Laser \& Photonics Rev. 1 231-248

[29] Zeytunyan A, et al. 2009 Nonlinear-dispersive similariton of passive fiber J. Europ. Opt. Soc. Rap. Public. 409009

[30] Iakushev S O, Shulika O V, and Sukhoivanov I A 2012 Passive nonlinear reshaping towards parabolic pulses in the steady-state regime in optical fibers Opt. Commun.

[31] Burgoyne B, Godbout N, and Lacroix S 2007 Nonlinear pulse propagation in optical fibers using second order moments Opt. Express 15 10075-10090

[32] Rosenberg C-K, et al. 2007 Evolution of optical pulses towards wave breaking in highly nonlinear fibres Opt. Commun. 273 272-277

[33] Kruglov V I, et al. 2000 Self-similar propagation of high-power parabolic pulses in optical fiber amplifiers Opt. Lett. 25 1753-1755

[34] Zhang J-F, Wu L, and Li L 2008 Self-similar parabolic pulses in optical fiber amplifiers with gain dispersion and gain saturation Phys. Rev. A 055801

[35] Kruglov V I, Aguergaray C, and Harvey J D 2012 Parabolic and hyper-Gaussian similaritons in fiber amplifiers and lasers with gain saturation Opt. Express 20 8741-8754

[36] Wang H, et al. 2010 Generation of triangular-shaped optical pulses in normally dispersive fibre $J$. Opt. 12035205

[37] Kalashyan M A, et al. 2010 Generation of transform-limited rectangular pulses in a spectral compressor Quantum Electronics 40868 